\title{
Resting-state networks in healthy adult subjects: a comparison between a 32-element and an 8-element phased array head coil at 3.0 Tesla
}

Acta Radiologica

20I5, Vol. 56(5) 605-613

(C) The Foundation Acta Radiologica 2015

Reprints and permissions:

sagepub.co.uk/journalsPermissions.nav DOI: $10.1|77 / 0284| 85 \mid$ | 4567703 acr.sagepub.com

@SAGE

\author{
Marco Paolini', Daniel Keeser ${ }^{\text {I,2 }}$, Michael Ingrisch', \\ Natalie Werner ${ }^{3}$, Nicole Kindermann ${ }^{3}$, Maximilian Reiser' ${ }^{\prime}$ and \\ Janusch Blautzik'
}

\begin{abstract}
Background: Little research exists on the influence of a magnetic resonance imaging (MRI) head coil's channel count on measured resting-state functional connectivity.

Purpose: To compare a 32-element (32ch) and an 8-element (8ch) phased array head coil with respect to their potential to detect functional connectivity within resting-state networks.

Material and Methods: Twenty-six healthy adults (mean age, 21.7 years; SD, 2.I years) underwent resting-state functional MRI at 3.0 Tesla with both coils using equal standard imaging parameters and a counterbalanced design. Independent component analysis (ICA) at different model orders and a dual regression approach were performed. Voxel-wise non-parametric statistical between-group contrasts were determined using permutation-based nonparametric inference.

Results: Phantom measurements demonstrated a generally higher image signal-to-noise ratio using the 32ch head coil. However, the results showed no significant differences between corresponding resting-state networks derived from both coils $(p<0.05$, FWE-corrected).

Conclusion: Using the identical standard acquisition parameters, the 32 ch head coil does not offer any significant advantages in detecting ICA-based functional connectivity within RSNs.
\end{abstract}

\section{Keywords}

CNS, magnetic resonance functional imaging, functional connectivity, equipment, phantom study, signal-to-noise ratio

Date received: 16 August 2013; accepted: 16 December 2014

\section{Introduction}

Functional magnetic resonance imaging (fMRI) enables the detection of functional connectivity within neural resting-state networks (RSNs) (1-4) that are known to be altered in various neuropsychiatric and neurodegenerative disorders (5-7). Despite a large amount of research in this field, data on the impact of different types of MRI head coils and different channel numbers for recording resting-state functional connectivity is still rare. Only one study has reported several advantages including an extended delineation of functional connections in the default mode network (DMN) for the use of a 32-element (32ch) phased array head coil compared to a 12-element (12ch) head coil (8). Others demonstrated benefits in terms of an improved

\footnotetext{
'Institute for Clinical Radiology, Ludwig-Maximilians-University Munich, Germany

${ }^{2}$ Department of Psychiatry and Psychotherapy, Ludwig-MaximiliansUniversity, Munich, Germany

${ }^{3}$ Department of Psychology, Ludwig-Maximilians-University, Munich, Germany
}

\section{Corresponding author:}

Marco Paolini, Institute for Clinical Radiology, Ludwig-MaximiliansUniversity Munich, Ziemssenstr. I, 80336 Munich, Germany.

Email: marco.paolini@med.uni-muenchen.de 
connectivity within the DMN for the use of an ultrahigh field of 7 Tesla in combination with a 16-element (16ch) head coil compared with a field strength of 3 Tesla in combination with an 8-element (8ch) head coil (9).

Generally, the use of multichannel phased array head coils has been shown to increase the image signal-to-noise ratio (SNR) (10-12) and to enable scan acceleration and artifact reduction via parallel imaging techniques $(13,14)$. Previous studies on the impact of higher field strengths (15) and higher channel count coils $(16,17)$ - not focusing on functional connectivity - revealed an increase in image SNR combined with a substantial gain in the blood-oxygen level dependent (BOLD)-signal or the time-series SNR (tSNR) using commonly applied sequence parameters. However, when image SNR is growing, the simultaneous elevation of fMRI measured BOLD-signal is limited, as physiological noise becomes the most defining factor in comparison with thermal noise (18-20).

The aim of the current study was to investigate the influence of a head coil's channel count on resting-state functional connectivity. We hypothesized that despite the expected increase in physiological noise, the use of a higher channel count would result in a higher image SNR and potentially more robust network connectivity.

\section{Material and Methods}

\section{Head coils}

We used a 32ch and an 8ch phased array head coil, both commercial products of Philips Healthcare, Hamburg, Germany (Fig. 1). The two coils were receiving-only devices and compatible with parallel imaging using Sensivity Encoding (32ch: maximal SENSE factor 8; 8ch: maximal SENSE factors 3, 3, and 2 for AP-, RL-, and FH-direction) and Contrast Level Appearance (CLEAR).

\section{Phantom studies}

For the phantom measurements, an American College of Radiology (ACR) magnetic resonance accreditation phantom (JM, Specialty Parts, San Diego, CA, USA) was positioned in the center of the respective head coil in a defined spatial orientation according to its marks as if it were a head. The same echo-planar gradientecho sequence was used for each head coil as described in the section "In vivo measurements" below.

For the determination of the image SNR, interfering trends of each voxel's signal intensity time-course were removed by fitting a second order polynomial and subtracting the first and second order terms (16). Subsequently, SNR maps were calculated by dividing the mean intensity value of each voxel's signal timecourse by its standard deviation and compared between both coils.

\section{Subjects}

Twenty-six right-handed healthy adults (15 women; mean age, 21.7 years; age range/SD, 18-30/2.1 years) participated in the study after giving their written informed consent. They were non-smokers, did not take any medication or drugs and were not allowed to consume alcohol, nicotine, or caffeine from the evening before the examination. The study protocol was in accordance with the Declaration of Helsinki and approved by the local ethics committee.
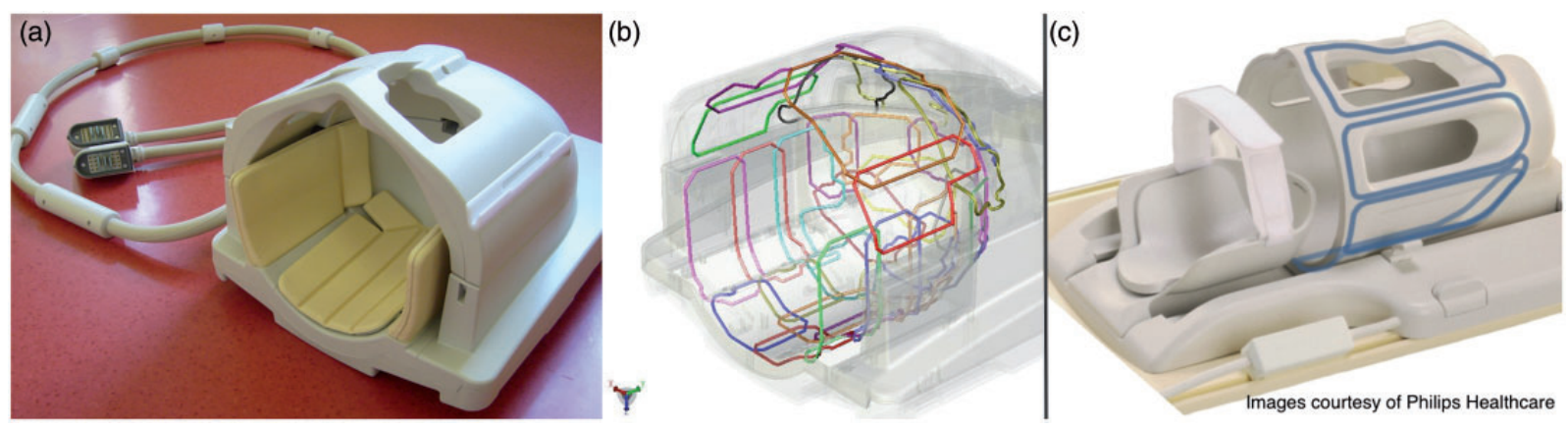

Fig. I. Designs of the $32 \mathrm{ch}$ and $8 \mathrm{ch}$ phased array head coils. (a) Picture of the $32 \mathrm{ch}$ phased array head coil showing the bottom section and the covering top portion. Both head coil parts can be disconnected. Internal dimensions based on the inferior opening: AP: $25 \mathrm{~cm}, \mathrm{RL}: 25 \mathrm{~cm}, \mathrm{FH}: 26 \mathrm{~cm}$. (b) Schematic image illustrating the circular arrangement of the 32 elements $(24$ elements are shown in the bottom part, 8 elements are shown in the top part). (c) Picture of the $8 \mathrm{ch}$ phased array head coil containing a superimposed schematic illustration of the annular arrangement of the coil elements (for a better overview, only 4 of the 8 elements of the one-piece coil are displayed). Internal dimensions based on the inferior opening: AP: $24 \mathrm{~cm}$, RL: $24 \mathrm{~cm}, \mathrm{FH}: 22 \mathrm{~cm}$. Images courtesy of Philips Healthcare, reprinted with permission. 


\section{In vivo measurements}

Imaging was performed using a 3.0 Tesla standard clinical scanner (Achieva TX, Philips Healthcare, Hamburg, Germany). For either head coil, functional data were acquired with a BOLD sensitive echo-planar gradient-echo sequence in axial orientation with the same imaging parameters: field of view (FOV), $230 \times 230 \times 132 \mathrm{~mm}$; voxel size, $3 \mathrm{~mm}$ isotropic; imaging matrix, $76 \times 77$; time of repetition (TR), $2500 \mathrm{~ms}$; time of echo (TE), $25 \mathrm{~ms}$; flip angle (FA), $90^{\circ}$; number of slices, 44; number of volumes, 180; SENSE, 1.8 ( $\mathrm{p}$ reduction, AP). Scans in both coils were acquired with an alternating, counterbalancing order to minimize any effects of different vigilance levels. Subjects were instructed not to move, not to fall asleep, to keep their eyes closed, and not to think of anything in particular. For anatomical reference, a high-resolution T1-weighted three-dimensional sequence was obtained in sagittal orientation using the $32 \mathrm{ch}$ head coil with the following imaging parameters: FOV, $240 \times 220 \times 200 \mathrm{~mm}$; voxel size, $1 \mathrm{~mm}$ isotropic; TR, $8.1 \mathrm{~ms}$; TE, $3.7 \mathrm{~ms}$; FA, $8^{\circ}$; number of slices, 200; SENSE, 2.5 (p reduction, AP)/2 (s reduction, RL). The total scan time was approximately $20 \mathrm{~min}$ per subject.

\section{Data preprocessing}

Anatomical and functional images were deobliqued, reoriented, and skull stripped using AFNI (Analysis of Functional NeuroImages, http://afni.nimh.nih.gov/ afni). The first five volumes of each functional scan were discarded for magnetic saturation effects. Further preprocessing of the functional data using FSL 4.1.7 (FMRIB Software Library, http:// www.fmrib.ox.ac.uk/fsl/index.html) included: realignment applying MCFLIRT (Motion Correction with FMRIB's Linear Image Registration Tool) (21), co-registration to the corresponding individual anatomical images, normalization to the MNI 152-template using FLIRT (FMRIB's Linear Image Registration Tool) version 5.5 (21) and spatial smoothing applying a $5 \mathrm{~mm}$ FWHM Gaussian kernel with high-pass temporal filtering (Gaussian-weighted least squares straight line fitting with sigma $=100 \mathrm{~s}$ ).

\section{Independent component analysis}

The data of all functional scans were combined in the MNI152 space (spatial concatenation) and reduced via Probabilistic Independent Component Analysis in FSL. Group-level component maps were computed based on data derived from all subjects and coils using FSL's Temporal Concatenation Group ICA (TC-GICA). This approach enabled the creation of corresponding
Independent Components (ICs) required for between-coil comparisons. The number of ICs was automatically estimated by FSL's MELODIC (Multivariate Exploratory Linear Optimized Decomposition into Independent Components) version 3.10. For further validation, we additionally performed analyses for the data derived from each coil separately as well as analyses for a predefined number of ICs using a low model order (20 ICs) (3) and a high model order (70 ICs) (22).

To be regarded as RSNs, connectivity patterns were required to extend over functionally relevant brain areas as described in previous studies (1-4) and to consist of characteristic ultra-slow frequencies in the BOLD-signal with a dominant peak in the range of $0.01-0.1 \mathrm{~Hz}(23-25)$. Connectivity patterns not conforming to these requirements were considered as artifacts.

To perform between-coil comparisons, the TC-GICA derived group-level ICs were reconstructed into individual ICs for each participant and each coil applying the dual regression approach $(1,3)$. Individual ICs were processed using voxel-wise non-parametric permutation testing (5000 permutations) with a Threshold-Free Cluster Enhancement (TFCE) permutation method (26) in order to generate averaged grouplevel components for both coils and between-coil contrasts of corresponding components in each direction. This method of comparison is mathematically restricted to the combined approach of both coils. The resulting maps were family-wise error (FWE) rate-controlled $(p<0.05)$.

The individual ICs generated in the dual regression approach were additionally used to obtain coil specific and subject specific fMRI time-courses for an inter-coil comparison on the single subject level. For each IC, the mean time-course of all voxels was normalized to z-values. Functional masks of the group analysis ICs (threshold, z-value $\geq 3$ ) were applied to exclude non-brain regions and low signal areas from the analysis. Mean z-values were calculated and compared by Wilcoxon signed rank test $(p<0.05$, corrected for multiple comparisons) using SPSS (IBM SPSS Statistics for Macintosh, Version 21.0. Armonk, NY, USA).

\section{Results}

\section{Phantom data}

As expected, the use of the 32ch coil resulted in a higher image SNR, both in regions near the phantom surface and hence close to the coil $(32 \mathrm{ch} / 8 \mathrm{ch}$ coil: Mean SNR $367 / 284$, SD $43 / 27$ ) as well as in central regions (32ch/ 8ch coil: Mean SNR 205/170, SD 14/14), shown in 
(a) 8 ch coil

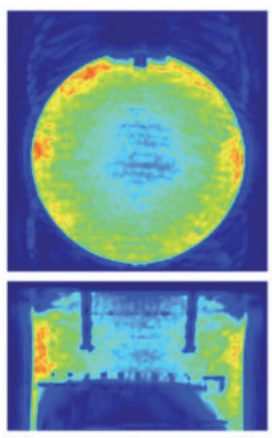

(b)

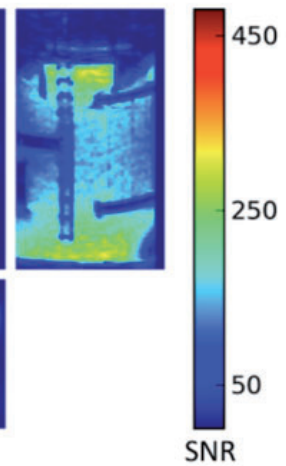

SNR
32 ch coil

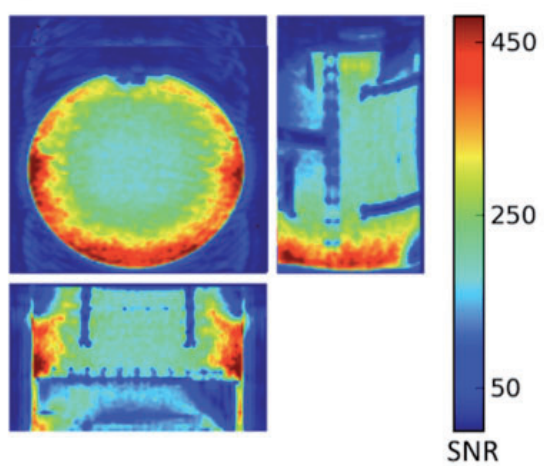

32 ch coil

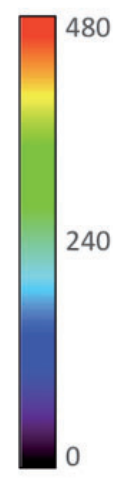

Fig. 2. Phantom measurements. (a) Image SNR maps of the $8 \mathrm{ch}$ (left illustration) and 32ch head coil (right illustration) generated by phantom measurements. (b) Extent of the peripheral and the central region of interest in comparable slices used for determination of mean SNR (right: $32 \mathrm{ch}$ coil, left: $8 \mathrm{ch}$ coil). Both maps use the same color scale.

Fig. 2. For both coils, no specific time drifts in SNR could be found in the regions of interest.

\section{Functional MRI data}

Using the MELODIC automatic dimensionality estimation, the TC-GICA approach yielded nine independent component maps for all subjects and coils including six RSNs (Fig. 3). Separately performed TC-GICA approaches for the $32 \mathrm{ch}$ and the $8 \mathrm{ch}$ coil showed the same number and types of ICs and RSNs, respectively.

The connectivity pattern represented in IC 1 corresponded to the classical DMN, mainly including the medial prefrontal cortex, the middle temporal gyrus, the precuneus cortex, the anterior and the posterior cingulate cortex, the thalamus as well as parahippocampal areas bilaterally (27). The connectivity pattern of IC 2 extended over brain regions related to autonomic and emotional processes including the ventromedial prefrontal cortex, the anterior and the posterior cingulate cortex, the insula, the basal ganglia, and the thalamus of both hemispheres (4). IC 3 was characterized by a connectivity pattern around the central sulci representing a sensorimotor network (28). A widespread connectivity pattern was represented in IC 4 containing different sensory subsystems including large parts of the auditory and visual cortex (4). IC 5 showed a connectivity pattern with a widely symmetrical fronto-temporo-parietal distribution known to be related to various cognitive operations $(3,4)$. IC 6 consisted of a connectivity pattern known to be associated with executive control extending over the prefrontal cortex, the anterior and middle cingulate cortex and the parieto-temporal junction bilaterally $(2,3)$. Finally, three ICs were regarded to represent artifacts caused by large vessels, cerebrospinal fluid pulsation, and head motion (IC 7, 8, and 9).

Additionally performed TC-GICA approaches for given numbers of ICs resulted in 13 RSNs (low model order ICA) and 43 RSNs (high model order ICA) for all subjects and coils.

By visual inspection, corresponding RSNs and corresponding artificial components recorded with either of the two head coils showed similar spatial extents, on the group level as well as on the single subject level (Fig. 4). Voxel-wise non-parametric statistics revealed 
(a)

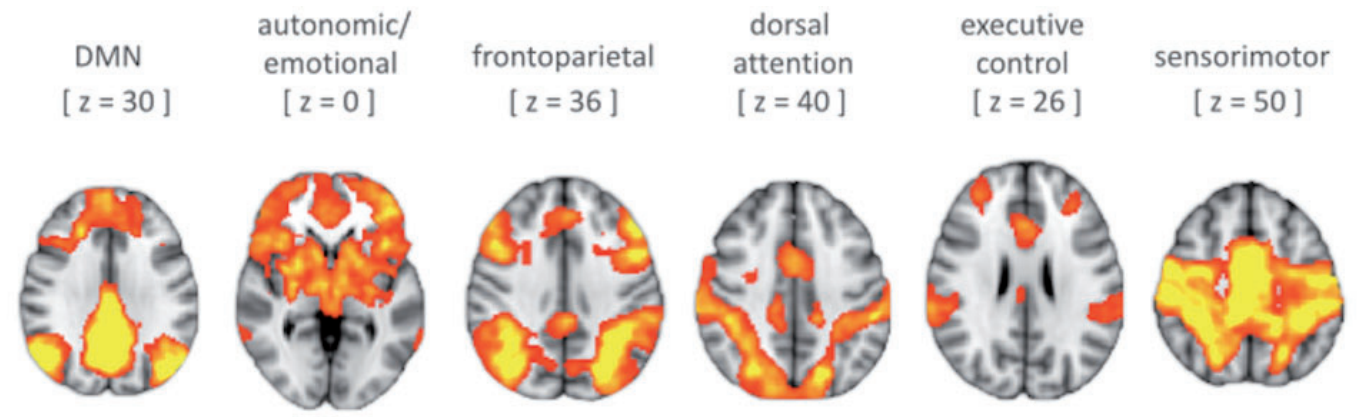

(b)
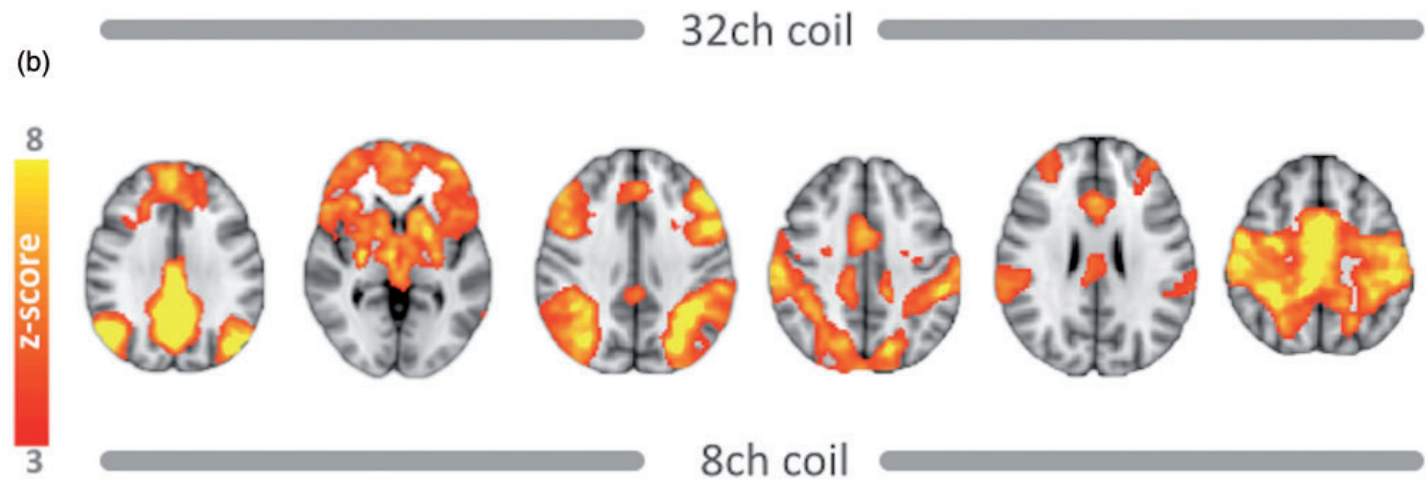

Fig. 3. RSNs acquired with the $32 \mathrm{ch}$ and the $8 \mathrm{ch}$ phased array head coil. Functional connectivity MRI networks shown for all 26 subjects and both coils as generated by the Temporal Concatenation Group ICA using a dual regression for an automatically estimated number of ICs (row a is showing the results of the $32 \mathrm{ch}$ coil, row b is showing the results of the $8 \mathrm{ch}$ coil). Functional connectivity patterns are superimposed on the MNII52-template. Axial images are displayed in radiological convention (z-coordinates in the MNII52 standard space are given in parenthesis).

no significant differences between the ICs of both groups in each direction ( $\mathrm{pFWE}<0.05$ ) for each combined TC-GICA approach. On the single subject level, the mean $\mathrm{z}$-values of corresponding ICs did not differ significantly between both head coils $\left(\mathrm{p}_{\text {corr }}<0.05\right)$. Detailed results of the single subject approach for the automatically estimated number of ICs are shown in Fig. 5.

\section{Discussion}

Here, we investigated the impact of a head coil's channel count on ICA-based resting-state functional connectivity by comparing a $32 \mathrm{ch}$ phased array head coil with a respective $8 \mathrm{ch}$ head coil in healthy adults. Statistical voxel-wise analyses revealed no significant differences between RSNs, and even artificial ICs, derived from the $32 \mathrm{ch}$ and the $8 \mathrm{ch}$ coil at different model orders demonstrating the reliability and reproducibility of functional connectivity across both coils. Even in the single subject analysis, no significant differences could be found. Especially the DMN and its subsystems known to be highly reliable and reproducible $(3,29,30)$ showed low intra-subject variability, i.e. a high degree of similarity between the results of the $32 \mathrm{ch}$ coil and the $8 \mathrm{ch}$ coil on the single subject level.
The hypothesis of more robust RSN connectivity patterns measured with a higher channel count head coil could not be verified in this experiment. However, our first consideration about a significant difference in SNR was confirmed by phantom measurements showing generally higher image SNR values for the 32ch head coil in comparison to the $8 \mathrm{ch}$ head coil.

In contrast to other studies, which reported less SNR gain or even a decrease in SNR in the center of higher channel count head coils $(12,16)$, we found a consistent increase in SNR regardless of the distance to the coil center. This finding may be caused by the fact that both coils have a multichannel phased array design and that the $32 \mathrm{ch}$ is well optimized regarding the arrangement of the additional elements despite the larger axial diameters. It is well known that compared to image SNR the BOLD-signal is limited by physiological noise, which affects time-series SNR (tSNR) $(13,18)$. Therefore, as physiological noise increases proportionally to the signal strength, the gain in tSNR is characterized by an asymptotic line with growing image SNR where physiological noise becomes the dominating confounder (18-20). Hence, it has been recommended to concentrate on higher spatial resolution or higher acceleration factors for greater benefits in tSNR (20). This could explain the results of our study since the 


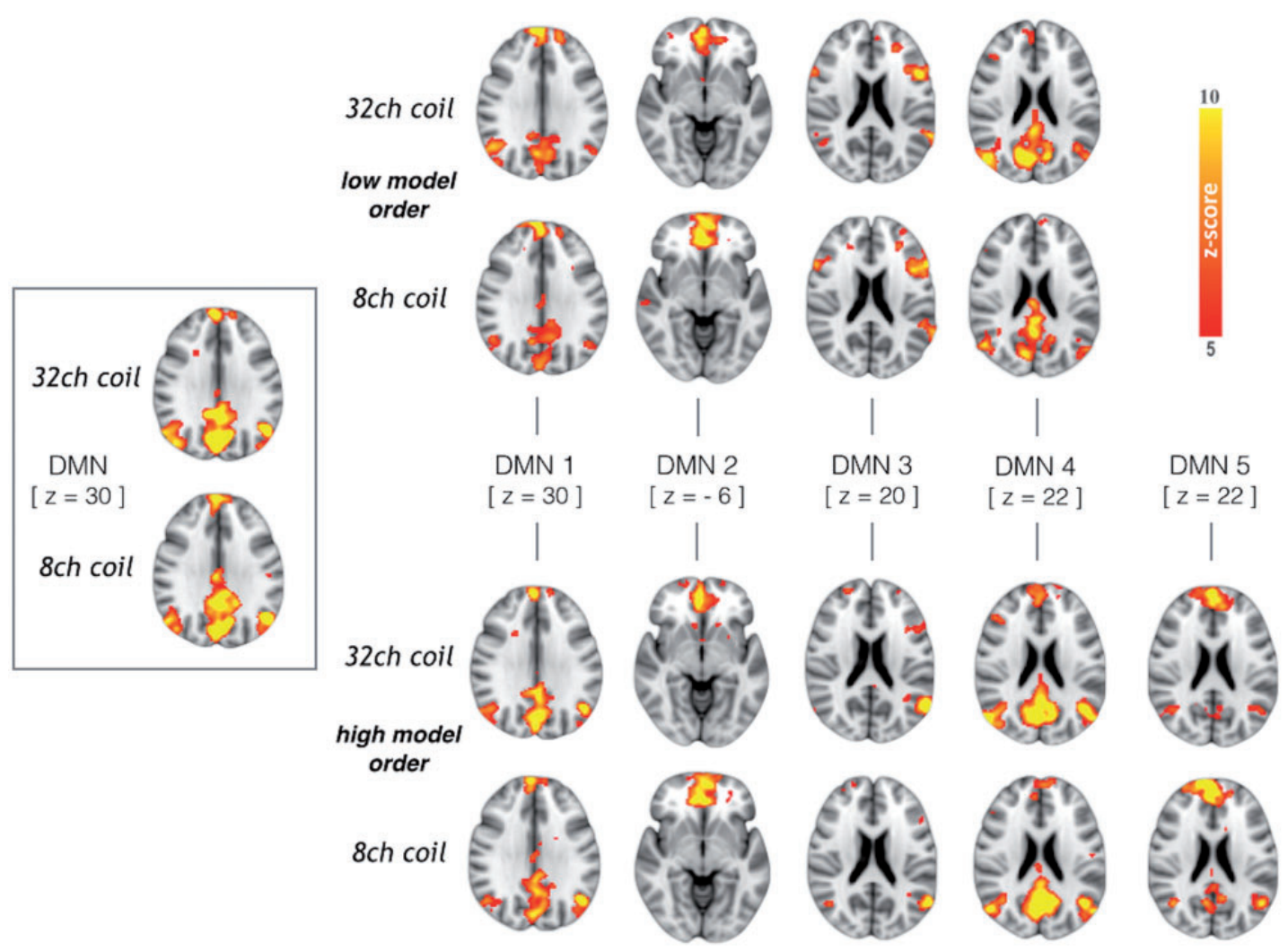

Fig. 4. Single subject data: The DMN and its subsystems (32ch vs. $8 \mathrm{ch}$ coil). Exemplary data of a single subject (subject 7) showing the default mode network of the automatically estimated model order ICA (left) and its subsystems generated by the low model order ICA and the high model order ICA (both right). Results of the $32 \mathrm{ch}$ and the $8 \mathrm{ch}$ head coil are compared. Functional connectivity patterns are superimposed on the MNII52-template. Axial images are displayed in radiological convention (z-coordinates in the MNII52 standard space are given in parenthesis).

unmodified sequence parameters may already have led to a relatively high image SNR sufficient for the reliable detection of RSNs using the 8ch phased array head coil. Nevertheless, in an fMRI experiment using a finger tapping task block design, Albrecht et al. (16) described advantages of a $32 \mathrm{ch}$ head coil compared to an $8 \mathrm{ch}$ head coil in terms of a significant increase in cortical activation in the motor and the somatosensory cortex applying standard sequence parameters. They attributed these findings to a higher image SNR. Other fMRI experiments with block design motor tasks also revealed more cortical activation when using head coils with a higher number of channels $(11,16,31)$. However, unlike paradigm driven fMRI activation studies, resting-state networks are usually detected in a low-frequency BOLD-signal range, which has been demonstrated to be strongly aliased with physiological noise deriving from cardiovascular and respiratory function that cannot be completely removed from the fMRI dataset $(32,33)$. The aliasing physiological noise in the fMRI dataset may act as a confounder, contributing to the lacking difference in the degree of RSN connectivity derived from either coil by decreasing tSNR.
To the best of our knowledge, the only study comparing RSNs recorded with different head coils was presented by Arnold et al. (8). In contrast to our study, they demonstrated advantages of a higher channel count head coil especially in terms of more robust functional connectivity in smaller nodes of the DMN. In our study the classical DMNs detected by the 32ch and $8 \mathrm{ch}$ coils both included the parahippocampal area bilaterally, which mainly accounted for the differences in the extent of the DMNs described by Arnold et al. (8). The inconsistent findings between both studies may be explained by different subject counts and statistical approaches given the higher participation (26 vs. 16 subjects) and the use of the more conservative FWE correction in our study that combined probably lead to more valid results. Moreover, also manufacturer-specific factors may have contributed to the different findings in each study. However, regardless of the limited comparability of both studies, one of the main reasons for the diverging results may be the differing sequence parameters, in particular the higher spatial resolution of the functional images in the study of Arnold et al., who used a voxel size of $2 \times 2 \times 2 \mathrm{~mm}^{3}$ 

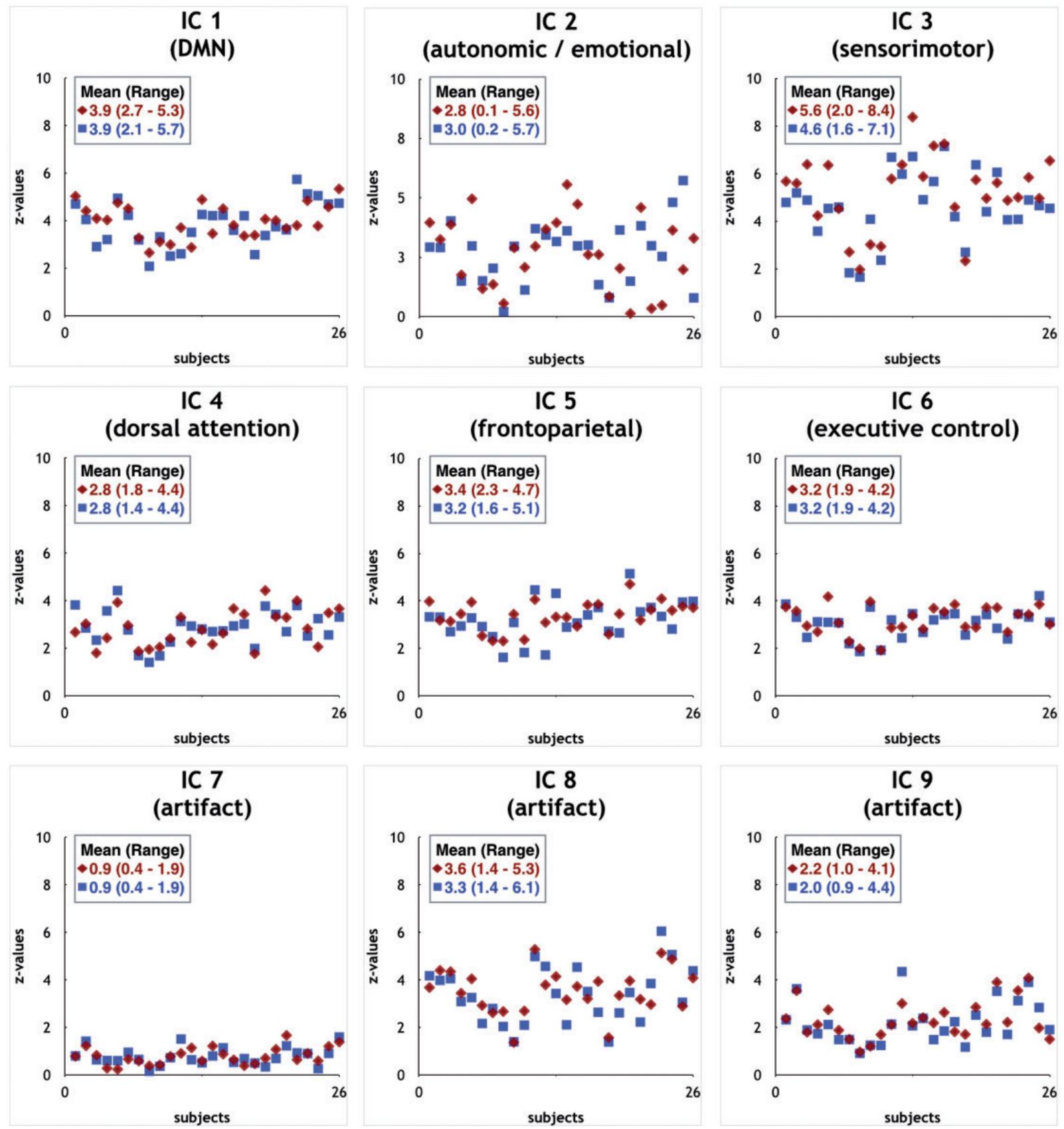

\section{$32 \mathrm{ch}$ coil $\quad 8 \mathrm{ch}$ coil}

Fig. 5. Mean z-values of the automatically estimated model order ICA. Illustration of the mean z-values of the single subject analysis based on the RSNs and the artificial ICs generated by the dual regression approach for an automatically estimated number of ICs. The results of the $32 \mathrm{ch}$ coil are directly compared to the results of the $8 \mathrm{ch}$ coil on the single subject level. The all over group-wise mean z-values and ranges of each IC are given in boxes.

compared to the standard voxel size of $3 \times 3 \times 3 \mathrm{~mm}^{3}$ used in our study.

As mentioned above, the decrease in image SNR at higher spatial resolution leads to a greater influence of thermal noise in comparison to physiological noise and hence to a greater benefit in BOLD-signal when using higher channel count head coils (20). For example,
Hale et al. (9) reported increased connectivity between DMN nodes in an ultra-high field utilizing a small voxel size of $1.5 \times 1.5 \times 3 \mathrm{~mm}^{3}$. Therefore, the most likely cause for the absence of an advantage in the detection of functional connectivity using the $32 \mathrm{ch}$ count coil seems to be the spatial resolution, even if the applied acceleration factor of 1.8 is also known to 
effect a beneficial decrease in the ratio of the physiological to thermal noise.

Our results cannot be simply generalized due to the dependency of signal strength on spatial resolution, acceleration factors, analysis methods, and coil design. The goal of our study was not to find certain sequence parameters that favor the use of the $32 \mathrm{ch}$ head coil. We consciously used standard sequence parameters in order to explore the potential use of both phased array head coils for detecting functional connectivity at common fMRI conditions.

Another limitation of the study is the indirect evidence of the similarity of both coils regarding resting-state functional connectivity by showing the lack of differences, i.e. accepting the null hypothesis. In this context, the decisive factor is the statistical power whose estimation depends on several parameters including the number of subjects, the percent BOLDsignal change and the intra- and inter-subject variability. In order to ensure a sufficiently high statistical power, we have chosen the number of subjects and the number of measured time points according to acknowledged recommendations for an fMRI study with a corrected $p$-value threshold (34), assuming the comparability of resting-state data and task-related data (2).

In conclusion, we could not find any significant advantage offered by the $32 \mathrm{ch}$ phased array receivingonly SENSE head coil regarding the robustness of ICA-based functional connectivity compared to a respective $8 \mathrm{ch}$ head coil when using the same standard sequence parameters. In order to take the best advantage of the higher image SNR provided by the 32ch head coil, SNR may be traded for a higher spatial resolution or a faster acquisition.

\section{Acknowledgements}

We thank Valerie Kirsch, Albert Berman, and Thomas Meindl for their work in producing this manuscript as well as Ruth Kreider for critically reading our manuscript.

\section{Conflict of interest}

None declared.

\section{Funding}

This research received no specific grant from any funding agency in the public, commercial, or not-for-profit sectors.

\section{References}

1. Biswal BB, Mennes M, Zuo XN, et al. Toward discovery science of human brain function. Proc Natl Acad Sci U S A 2010;107:4734-4739.

2. Damoiseaux JS, Rombouts SA, Barkhof F, et al. Consistent resting-state networks across healthy subjects. Proc Natl Acad Sci U S A 2006;103:13848-13853.
3. Zuo XN, Kelly C, Adelstein JS, et al. Reliable intrinsic connectivity networks: test-retest evaluation using ICA and dual regression approach. Neuroimage 2010;49: 2163-2177.

4. Laird AR, Fox PM, Eickhoff SB, et al. Behavioral interpretations of intrinsic connectivity networks. Cogn Neurosci 2011;23:4022-4037.

5. Greicius MD, Srivastava G, Reiss AL, et al. Defaultmode network activity distinguishes Alzheimer's disease from healthy aging: evidence from functional MRI. Proc Natl Acad Sci U S A 2004;101:4637-4642.

6. Rocca MA, Valsasina P, Absinta M, et al. Default-mode network dysfunction and cognitive impairment in progressive MS. Neurology 2010;74:1252-1259.

7. Stigler KA, McDonald BC, Anand A, et al. Structural and functional magnetic resonance imaging of autism spectrum disorders. Brain Res 2011;1380:146-161.

8. Arnold S, Whitfield-Gabrieli S, Shannon S, et al. Improved detection of functional connectivity MRI with 32-channel phased array head coil. Proc Intl Soc Mag Reson Med 2011;19:1637.

9. Hale JR, Brookes MJ, Hall EL, et al. Comparison of functional connectivity in default mode and sensorimotor networks at 3 and 7T. MAGMA 2010;23:339-349.

10. Roemer PB, Edelstein WA, Hayes CE, et al. The NMR phased array. Magn Reson Med 1990;16:192-225.

11. de Zwart JA, Ledden PJ, van Gelderen P, et al. Signalto-noise ratio and parallel imaging performance of a 16-channel receive-only brain coil array at 3.0 Tesla. Magn Reson Med 2004;51:22-26.

12. Wiggins GC, Triantafyllou $\mathrm{C}$, Potthast A, et al. 32-channel 3 Tesla receive-only phased-array head coil with soccer-ball element geometry. Magn Reson Med 2006;56:216-223.

13. de Zwart JA, van Gelderen P, Kellman P, et al. Application of sensitivity-encoded echo-planar imaging for blood oxygen level-dependent functional brain imaging. Magn Reson Med 2002;48:1011-1020.

14. Golay X, de Zwart JA, Ho YC, et al. Parallel imaging techniques in functional MRI. Top Magn Reson Imaging 2004;15:255-265.

15. Meindl T, Born C, Britsch S, et al. Functional BOLD MRI: comparison of different field strengths in a motor task. Eur Radiol 2008;18:1102-1113.

16. Albrecht J, Burke M, Haegler K, et al. Potential impact of a 32-channel receiving head coil technology on the results of a functional MRI paradigm. Clin Neuroradiol 2010;20:223-229.

17. Fellner C, Doenitz C, Finkenzeller T, et al. Improving the spatial accuracy in functional magnetic resonance imaging (fMRI) based on the blood oxygenation level dependent (BOLD) effect: benefits from parallel imaging and a 32-channel head array coil at 1.5 Tesla. Clin Hemorheol Microcirc 2009;43:71-82.

18. Kruger G, Glover GH. Physiological noise in oxygenation-sensitive magnetic resonance imaging. Magn Reson Med 2001;46:631-637.

19. Triantafyllou C, Hoge RD, Krueger G, et al. Comparison of physiological noise at $1.5 \mathrm{~T}, 3 \mathrm{~T}$ and $7 \mathrm{~T}$ and 
optimization of fMRI acquisition parameters. Neuroimage 2005;26:243-250.

20. Triantafyllou C, Polimeni JR, Wald LL. Physiological noise and signal-to-noise ratio in fMRI with multichannel array coils. Neuroimage 2011;55:597-606.

21. Jenkinson M, Bannister P, Brady $\mathbf{M}$, et al. Improved optimization for the robust and accurate linear registration and motion correction of brain images. Neuroimage 2002; 17:825-841.

22. Kiviniemi V, Starck T, Remes J, et al. Functional segmentation of the brain cortex using high model order group PICA. Hum Brain Mapp 2009;30:3865-3886.

23. Fox MD, Snyder AZ, Vincent JL, et al. The human brain is intrinsically organized into dynamic, anticorrelated functional networks. Proc Natl Acad Sci U S A 2005; 102:9673-9678.

24. Vincent JL, Patel GH, Fox MD, et al. Intrinsic functional architecture in the anaesthetized monkey brain. Nature 2007;447:83-86.

25. Boly M, Phillips C, Balteau E, et al. Consciousness and cerebral baseline activity fluctuations. Hum Brain Mapp 2008;29:868-874.

26. Smith SM, Nichols TE. Threshold-free cluster enhancement: addressing problems of smoothing, threshold dependence and localisation in cluster inference. Neuroimage 2009;44:83-98.

27. Raichle ME, MacLeod AM, Snyder AZ, et al. A default mode of brain function. Proc Natl Acad Sci U S A 2001; 98:676-682.
28. Biswal B, Yetkin FZ, Haughton VM, et al. Functional connectivity in the motor cortex of resting human brain using echo-planar MRI. Magn Reson Med 1995;34: 537-541.

29. Meindl T, Teipel S, Elmouden R, et al. Test-retest reproducibility of the default-mode network in healthy individuals. Hum Brain Mapp 2010;31:237-246.

30. Franco AR, Pritchard A, Calhoun VD, et al. Interrater and intermethod reliability of default mode network selection. Hum Brain Mapp 2009;30:2293-2303.

31. Kaza E, Klose U, Lotze M. Comparison of a 32-channel with a 12-channel head coil: are there relevant improvements for functional imaging? J Magn Reson Imaging 2011;34:173-183.

32. Birn RM, Murphy K, Bandettini PA. The effect of respiration variations on independent component analysis results of resting state functional connectivity. Hum Brain Mapp 2008;29:740-750.

33. Starck T, Remes J, Nikkinen J, et al. Correction of lowfrequency physiological noise from the resting state BOLD fMRI-Effect on ICA default mode analysis at 1.5 T. J Neurosci Methods 2010;186:179-185.

34. Desmond JE, Glover GH. Estimating sample size in functional MRI (fMRI) neuroimaging studies: statistical power analyses. J Neurosci Methods 2002;118: 115-128. 